\title{
Gomory-Hu trees of infinite graphs with finite total weight
}

\author{
Attila Joó *
}

2016

\begin{abstract}
Gomory and $\mathrm{Hu}$ proved in [3] their well-known theorem which states that if $G$ is a finite graph with non-negative weights on its edges, then there exists a tree $T$ (called now GomoryHu tree) on $V(G)$ such that for all $u \neq v \in V(G)$ there is an $e \in E(T)$ such that the two components of $T-e$ determines an optimal (minimal valued) cut between $u$ an $v$ in $G$. In this paper we extend their result to infinite weighted graphs with finite total weight. Furthermore, we show by an example that one can not omit the condition of finiteness of the total weight.
\end{abstract}

\section{Introduction}

Let $G=(V, E)$ be a countable connected simple graph and let $c: E \rightarrow \mathbb{R}_{+} \backslash\{0\}$ be a weightfunction, then $(V, E, c)$ is a weighted graph. We call the subsets $X$ of $V$ cuts and we write out $_{G}(\boldsymbol{X})$ for the set of the edges with exactly one end in $X$. We say $X$ is an $\boldsymbol{u}-\boldsymbol{v}$ cut for some $u \neq v \in V$ if $u \in X$ and $v \notin X$. A cut $X$ separates $u$ and $v$ if $X$ is either a $u-v$ or a $v-u$ cut. Let $\boldsymbol{d}_{\boldsymbol{c}}(\boldsymbol{X})=\sum_{e \in \mathrm{out}_{G}(X)} c(e)$ and let $\boldsymbol{\lambda}_{\boldsymbol{c}}(\boldsymbol{u}, \boldsymbol{v}):=\inf \left\{d_{c}(X): X\right.$ is a $u-v$ cut $\}$ for $u \neq v \in V$. A cut $X$ is an optimal $\boldsymbol{u}-\boldsymbol{v}$ cut if it is a $u-v$ cut with $d_{c}(X)=\lambda_{c}(u, v)$. A cut $X$ is optimal if it is an optimal $u-v$ cut for some $u \neq v \in V$. The weighted graph $(V, E, c)$ is finitely separable if $\lambda_{c}$ has just finite values. A tree $T=(V, F)$ is a Gomory-Hu tree for $(V, E, c)$ if for all $u \neq v \in V$ there is an $e \in F$ such that the fundamental cuts corresponding to $e$ (i.e. the vertex sets of the components of $T-e)$ separate optimally $u$ and $v$ in $(V, E, c)$. Gomory and Hu proved in [3] that for all finite weighted graph there exists a Gomory-Hu tree. It has several interesting consequences. For example the function $\lambda_{c}$ may have at most $n-1$ different values instead of $\left(\begin{array}{l}n \\ 2\end{array}\right)$ (where $n$ is the number of the vertices) and there is at least two optimal cuts that consist of a single vertex, namely the leafs of the Gomory-Hu tree (unless the graph is trivial).

In this paper we extend their theorem for infinite weighted graphs with finite total weight. Note that, the strict positivity of $c$ and the connectedness of $G$ are not real restrictions since throwing away edges $e$ with $c(e)=0$ has no effect on the values of the cuts and one can construct Gomory-Hu trees component-wise and join them to a Gomory-Hu tree. Furthermore, if the sum of the weights is finite, then the weighted graph must be countable.

The cut structure of infinite graphs has been already investigated in some other perspectives (see for example [2] and [1]) where the authors only allow cuts with finitely many outgoing edges. As it seems from the definitions above we are focusing on the literal generalization of Gomory-Hu

*MTA-ELTE Egerváry Research Group, Department of Operations Research, Eötvös Loránd University, Budapest, Hungary. Email: joapaat@cs.elte.hu 
trees. In a more abstract folklore version of the Gomory-Hu theorem there is no graph, one just has a finite set $V$ and a function $b: \mathcal{P}(V) \rightarrow \mathbb{R}_{+}$which is symmetric $(b(X)=b(V \backslash X))$ and submodular i.e.

$$
b(X)+b(Y) \geq b(X \cap Y)+b(X \cup Y) \text { if } X, Y \subseteq V .
$$

Let $\lambda_{b}(u, v)=\inf \{b(X): X$ a $u-v$ cut $\}$ (this definition makes sense for infinite $V$ as well). In this case, there exists an abstract Gomory-Hu tree with respect to $b$ in the following sense. There is a tree $T$ on the vertex set $V$ in such a way that for every $u \neq v \in V$ there is some $e \in E(T)$ such that for a fundamental cut $X$ corresponding to $e$, we have $b(X)=\lambda_{b}(u, v)$.

\section{Preparations}

Let $(V, E, c)$ be a weighted graph.

Proposition 1. $d_{c}(X)+d_{c}(Y) \geq d_{c}(X \cup Y)+d_{c}(X \cap Y)$ for all $X, Y \subseteq V$.

Proof: If edge $e$ goes between $X \backslash Y$ and $Y \backslash X$, then it contributes $2 c(e)$ to the left side and 0 to the right side of the inequality. The contribution of any other type of edge is the same for both sides.

For a sequence $\left(X_{n}\right)$ let

$$
\begin{aligned}
& \liminf X_{n}=\bigcup_{m=0}^{\infty} \bigcap_{n=m}^{\infty} X_{n} \\
& \limsup X_{n}=\bigcap_{m=0}^{\infty} \bigcup_{n=m}^{\infty} X_{n} .
\end{aligned}
$$

If $\lim \inf X_{n}=\lim \sup X_{n}$, then we denote this set by $\lim X_{n}$ and we say that $\left(X_{n}\right)$ is convergent.

\section{Claim 2.}

1. If $\left(X_{n}\right)$ is a convergent sequence of cuts, then $d_{c}\left(\lim X_{n}\right) \leq \liminf d_{c}\left(X_{n}\right)$.

2. In addition, if $\sum_{e \in E} c(e)<\infty$, then $\lim d_{c}\left(X_{n}\right)$ exists and $\lim d_{c}\left(X_{n}\right)=d_{c}\left(\lim X_{n}\right)$ holds.

Proof: It is routine to check that out ${ }_{G}\left(\lim X_{n}\right)=\operatorname{limout}_{G}\left(X_{n}\right)$ holds. Consider the discrete measure space $(E, \mathcal{P}(E), \tilde{c})$ where $\tilde{c}(F)=\sum_{e \in F} c(e)$ for $F \subseteq E$.

By applying the Fatou lemma to the characteristic functions of the $\operatorname{sets} \operatorname{out}_{G}\left(X_{n}\right)$ we obtain

$$
\begin{aligned}
& d_{c}\left(\lim \left(X_{n}\right)\right)=\widetilde{c}\left(\operatorname{out}_{G}\left(\lim X_{n}\right)\right)=\widetilde{c}\left(\lim \operatorname{out}_{G}\left(X_{n}\right)\right)= \\
& \widetilde{c}\left(\liminf \operatorname{out}_{G}\left(X_{n}\right)\right) \leq \liminf \widetilde{c}\left(\text { out }_{G}\left(X_{n}\right)\right)=\liminf d_{c}\left(X_{n}\right) .
\end{aligned}
$$

At the statement 2 we have $\widetilde{c}(E)<\infty$, thus the constant 1 function is integrable. Therefore by using Lebesgue theorem to the characteristic functions of the sets out ${ }_{G}\left(X_{n}\right)$ we obtain

$$
d_{c}\left(\lim X_{n}\right)=\widetilde{c}\left(\lim \operatorname{out}_{G}\left(X_{n}\right)\right)=\lim \widetilde{c}\left(\operatorname{out}_{G}\left(X_{n}\right)\right)=\lim d_{c}\left(X_{n}\right) .
$$

Let us formulate our main result in the following more abstract way. 
Theorem 3. Let $V$ be a nonempty countable set and let $b: \mathcal{P}(V) \rightarrow \mathbb{R}_{+} \cup\{\infty\}$ such that

0. $b(X)=0 \Longleftrightarrow X \in\{\varnothing, V\}$,

1. $b(X)=b(V \backslash X)$ for $X \subseteq V$, (b is symmetric)

2. $b(X)+b(Y) \geq b(X \cap Y)+b(X \cup Y)$ for $X, Y \subseteq V$, (b is submodular)

3. if $\left(X_{n}\right)$ is a nested sequence of cuts, then $b\left(\lim X_{n}\right)=\lim b\left(X_{n}\right)$ (b is monotone-continuous)

4. $\lambda_{b}$ has only finite values. (b finitely separate)

Then there exists an abstract Gomory-Hu tree with respect to b.

Remark 4. Properties 1,2 imply that for any $X, Y$ we also have

$$
b(X)+b(Y) \geq b(X \backslash Y)+b(Y \backslash X) .
$$

Observe that if $\sum_{e \in E} c(e)<\infty$ holds, then $b:=d_{c}$ satisfies the properties above. Hence as a special case of Theorem 3 we obtain:

Corollary 5. Every weighted graph with $\sum_{e \in E} c(e)<\infty$ admits a Gomory-Hu tree.

Consider the following weakening of 3 .

$3^{\prime}$ if $\left(X_{n}\right)$ is a nested sequence of cuts, then $b\left(\lim X_{n}\right) \leq \liminf b\left(X_{n}\right)$.

If we do not assume $\sum_{e \in E} c(e)<\infty$ and we demand just $(V, E, c)$ to be finitely separable, then Claim 2 ensures that $b:=d_{c}$ still satisfy this weaker condition (see Claim 2/1). We will see by a counterexample that in this case one can not guarantee the existence of a Gomory-Hu tree. Even so, the next theorem provides something similar but weaker. A system of sets is called laminar if any two members of it are either disjoint or $\subseteq$-comparable.

Theorem 6. If b satisfies conditions 0,1,2,3',4, then there is a laminar system $\mathcal{L}^{*}$ of optimal cuts such that any pair from $V$ is separated optimally by some element of $\mathcal{L}^{*}$.

Proof:

Claim 7. For any $u \neq v \in V$ there exists an $u-v$ cut $X^{*}$ with $b\left(X^{*}\right)=\lambda_{b}(u, v)$.

Proof: Let $u, v$ be fix. The error of the sequence $\left(X_{n}\right)$ of $u-v$ cuts is

$$
\sum_{n=0}^{\infty}\left(b\left(X_{n}\right)-\lambda(u, v)\right)
$$

It is enough to prove the existence of a nested sequence $\left(Y_{n}\right)$ of $u-v$ cuts with finite error. Indeed, from the finiteness of the error it follows that $\lim b\left(Y_{n}\right)=\lambda(u, v)$, hence by property 3 '

$$
\lambda_{b}(u, v) \leq b\left(\bigcap_{n=0}^{\infty} Y_{n}\right) \leq \liminf b\left(Y_{n}\right)=\lim b\left(Y_{n}\right)=\lambda_{b}(u, v) .
$$


Proposition 8. For any sequence $\left(X_{n}\right)$ with finite error there is another sequence $\left(Z_{n}\right)$ with less or equal error such that $Z_{0} \supseteq \bigcup_{n=1}^{\infty} Z_{n}$.

Proof: Replace in the sequence $\left(X_{n}\right)$ the member $X_{0}$ by $X_{0} \cup X_{1}$ and the member $X_{1}$ by $X_{1} \cap X_{0}$. By submodularity the error of the new sequence $\left(X_{n}^{1}\right)$ is less or equal. Then replace $X_{0}^{1}=X_{0} \cup X_{1}$ by $X_{0}^{2}:=X_{0}^{1} \cup X_{2}^{1}=X_{0} \cup X_{1} \cup X_{2}$ and replace $X_{2}^{1}$ by $X_{2}^{2}:=X_{2}^{2} \cap X_{0}^{2}=X_{2} \cap\left(X_{0} \cup X_{1}\right)$. In general let

$$
X_{n}^{m+1}= \begin{cases}X_{0}^{m} \cup X_{m+1}^{m} & \text { if } n=0 \\ X_{0}^{m} \cap X_{m+1}^{m} & \text { if } n=m+1 \\ X_{n}^{m} & \text { otherwise. }\end{cases}
$$

Finally we claim that the following "limit" of these sequences is appropriate.

$$
\begin{aligned}
& Z_{0}:=\bigcup_{n=0}^{\infty} X_{n} \\
& Z_{n+1}:=X_{n+1} \cap\left(\bigcup_{i=0}^{n} X_{n}\right) .
\end{aligned}
$$

For

$$
S_{m}:=\sum_{n=0}^{\infty}\left(b\left(X_{n}^{m}\right)-\lambda(u, v)\right)
$$

$\left(S_{m}\right)$ is a non-negative decreasing sequence thus it has a limit $S$ i.e.

$$
S=\lim _{m \rightarrow \infty} \sum_{n=0}^{\infty}\left(b\left(X_{n}^{m}\right)-\lambda(u, v)\right) .
$$

Consider the counting measure on $\mathbb{N}$ and apply Fatou lemma:

$$
\begin{aligned}
S & =\liminf _{m} \sum_{n=0}^{\infty}\left(b\left(X_{n}^{m}\right)-\lambda(u, v)\right) \\
& \geq \sum_{n=0}^{\infty}\left(\liminf _{m} b\left(X_{n}^{m}\right)-\lambda(u, v)\right) \\
& =\liminf _{m} b\left(\bigcup_{i=0}^{m} X_{i}\right)-\lambda(u, v)+\sum_{n=1}^{\infty}\left(b\left(Z_{n}\right)-\lambda(u, v)\right) \\
& \geq \sum_{n=0}^{\infty}\left(b\left(Z_{n}\right)-\lambda(u, v)\right) .
\end{aligned}
$$

At the last step we applied property $3^{\prime}\left(S_{0}+\lambda(u, v)\right.$ is an obvious upper bound for $\left\{b\left(X_{0}^{m}\right)\right.$ : $m \in \mathbb{N}\})$. Hence the error of $\left(Z_{n}\right)$ is smaller or equal than the error of the earlier sequences.

Let $X_{n}$ be a $u-v$ cut with $b\left(X_{n}\right)-\lambda(u, v) \leq 1 / 2^{n+1}$. Then the error of $\left(X_{n}\right)$ is at most 1 . Apply Proposition 8 with $\left(X_{n}\right)$ to obtain $\left(X_{n}^{1}\right)$ and let $Y_{0}=X_{0}^{1}$. Use Proposition 8 to the terminal segment 
of $\left(X_{n}^{1}\right)$ consists all but the 0 -th element (this sequence has error at most $\left.1-\left(b\left(Y_{0}\right)-\lambda_{b}(u, v)\right)\right)$ to obtain $\left(X_{n}^{2}\right)$ and let $Y_{1}=X_{0}^{2}$. By continuing the process recursively we build up a desired nested $\left(Y_{n}\right)$ with error at most 1.

Remark 9. One can observe in the proof above that if $\left(X_{n}\right)$ is a sequence of $u-v$ cuts with finite error, then simply $\bigcup_{n=0}^{\infty} X_{n}$ and $\bigcap_{n=0}^{\infty} X_{n}$ are optimal $u-v$ cuts.

Proposition 10. The intersection and the union of (even infinitely many) optimal $u-v$ cuts is an optimal $u-v$ cut.

Proof: Let $X$ and $Y$ be optimal $u-v$ cuts. On the one hand, $b(X) \leq b(X \cup Y)$ and $b(Y) \leq b(X \cap Y)$ hold since $X \cup Y$ and $X \cap Y$ are $u-v$ cuts. Thus

$$
b(X)+b(Y) \leq b(X \cup Y)+b(X \cap Y) .
$$

On the other hand,

$$
b(X)+b(Y) \geq b(X \cup Y)+b(X \cap Y)
$$

by submodularity. Hence equality holds and therefore $b(X)=b(X \cup Y)$ and $b(Y)=b(X \cap Y)$ since $b(X), b(Y)<\infty$ because of property 4 . By induction we know the statement for finitely many optimal $u-v$ cuts. Consider an infinite family $\mathcal{X}$ of optimal $u-v$ cuts. Let $V=\left\{v_{n}: n \in \mathbb{N}\right\}$ and let $X_{n}^{\prime} \in \mathcal{X}$ with $v_{n} \notin X_{n}^{\prime}$ if $v_{n} \notin \bigcap \mathcal{X}$ and an arbitrary element of $\mathcal{X}$ otherwise. Then $X_{n}:=\bigcap_{m=0}^{n} X_{m}^{\prime}$ is an optimal $u-v$ cut again and $\bigcap_{n=0}^{\infty} X_{n}=\bigcap \mathcal{X}$ as well by property 3 '.

Corollary 11. There is $a \subseteq$-smallest (largest) optimal $u-v$ cut $\boldsymbol{X}_{\boldsymbol{u}, \boldsymbol{v}}\left(Y_{u, v}\right)$ which is the intersection (union) of all optimal $u-v$ cuts.

Claim 12. Let $X$ be an optimal $s-t$ cut and let $Y$ be an optimal $u-v$ cut.

1. Assume $X$ is a $u-v$ cut. Then $Y \cup X$ is an optimal $u-v$ cut if $t \notin Y$ and $Y \cap X$ is an optimal $u-v$ cut if $t \in Y$.

2. Assume $X$ is a $v-u$ cut. Then $Y \cup(V \backslash X)$ is an optimal $u-v$ cut if $s \notin Y$ and $Y \backslash X$ is an optimal $u-v$ cut if $s \in Y$.

3. Assume $u, v \in X$. Then $Y \cap X$ is an optimal $u-v$ cut if $t \notin Y$ and $Y \cup(V \backslash X)$ is an optimal $u-v$ cut if $t \in Y$.

4. Assume $u, v \notin X$. Then $Y \backslash X$ is an optimal $u-v$ cut if $s \notin Y$ and $Y \cup X$ is an optimal $u-v$ cut if $s \in Y$.

Proof: It is enough to prove 1 and 3 since by replacing $X$ with the optimal $t-s$ cut $V \backslash X$ in them we obtain 2 and 4 respectively. To prove 1 assume first that $t \notin Y$. Since $X \cup Y$ is a $s-t$ cut and $X \cap Y$ is a $u-v$ cut we have $b(X \cup Y) \geq b(X)$ and $b(X \cap Y) \geq b(Y)$. Combining this with submodularity we get

$$
b(X)+b(Y) \geq b(X \cup Y)+b(X \cap Y) \geq b(X)+b(Y),
$$

thus equality must hold in both inequalities. If $t \in Y$ and $s \in Y$, then $X \cup Y$ is a $u-v$ cut and $X \cap Y$ is a $s-t$ cut; therefore by arguing similarly as above we obtain that $X \cup Y$ must be an optimal $u-v$ cut. Finally if $t \in Y$ and $s \notin Y$, then on the one hand $Y$ separates $t$ and $s$ and $X$ does this optimally therefore $b(X) \leq b(Y)$, on the other hand $Y$ is an optimal $u-v$ cut and $X$ is an $u-v$ cut hence $b(Y) \leq b(X)$. Thus $b(X)=b(Y)$ therefore $X$ and $Y$ both are optimal $u-v$ cuts hence by Proposition $10 X \cup Y$ and $X \cap Y$ as well. The proof of 3 is similar. 
Corollary 13. If $X$ is an optimal $s-t$ cut and $u \neq v \in X$, then either $X_{u, v} \subseteq X$ or $X_{v, u} \subseteq X$ (where $X_{x, y}$ stands for the $\subseteq$-smallest optimal $x-y$ cut).

Proof: If $X_{u, v} \subseteq X$, then we are done. Assume $X_{u, v} \nsubseteq X$. By the minimality of $X_{u, v}$ the $u-v$ cut $X_{u, v} \cap X$ cannot be optimal therefore by Claim $12 / 3 X_{u, v} \cup(V \backslash X)$ is an optimal $u-v$ cut. But then $V \backslash\left[X_{u, v} \cup(V \backslash X)\right]=X \backslash X_{u, v}$ is an optimal $v-u$ cut therefore we obtain $X_{v, u} \subseteq\left(X \backslash X_{u, v}\right) \subseteq X$.

Theorem 6 follows immediately from the next lemma (actually we need the lemma just with finite $\mathcal{L})$.

Lemma 14. If $\mathcal{L}$ is a laminar system of optimal cuts and $u \neq v \in V$, then there is a cut $X^{*}$ for which $\mathcal{L} \cup\left\{X^{*}\right\}$ is laminar and $X^{*}$ separates optimally $u$ and $v$.

Proof: Let us partition $\mathcal{L}$ into four parts $\mathcal{L}_{u, \bar{v}}:=\{X \in \mathcal{L}: u \in X \wedge v \notin X\}$, we define $\mathcal{L}_{\bar{u}, \boldsymbol{v}}, \mathcal{L}_{\boldsymbol{u}, \boldsymbol{v}}$ and $\mathcal{L}_{\bar{u}, \bar{v}}$ similarly. If $X_{u, v} \subseteq \widehat{X}$ for some $\widehat{X} \in \mathcal{L}_{u, \bar{v}}$, then $\left\{X_{u, v}\right\} \cup \mathcal{L}_{u, v} \cup \mathcal{L}_{\bar{u}, v}$ is laminar. Suppose that we have no such an $\widehat{X}$ not even if we interchange $u$ and $v$. By Corollary 13 we know that for all $W \in \mathcal{L}_{u, v}$ either $X_{u, v} \subseteq W$ or $X_{v, u} \subseteq W$. Hence by symmetry we may assume that $\bigcap \mathcal{L}_{u, v} \supseteq X_{u, v}$. We will show that $\left\{X_{u, v}\right\} \cup \mathcal{L}_{u, v} \cup \mathcal{L}_{\bar{u}, v}$ is laminar in this case as well. Let $X \in \mathcal{L}_{\bar{u}, v}$ be arbitrary. Then $X_{v, u} \nsubseteq \subseteq X$ otherwise $\widehat{X}:=X$ would be a bound. But then $X_{v, u} \cap X$ cannot be an optimal $v-u$ cut by the minimality of $X_{v, u}$. Therefore by Claim 12/1 we know that $X_{v, u} \cup X$ is an optimal $v-u$ cut and hence $V \backslash\left(X_{v, u} \cup X\right)$ is an optimal $u-v$ cut. Thus $V \backslash\left(X_{v, u} \cup X\right) \supseteq X_{u, v}$ from which $X \cap X_{u, v}=\varnothing$ follows.

Thus we may suppose that $\left\{X_{u, v}\right\} \cup \mathcal{L}_{u, v} \cup \mathcal{L}_{\bar{u}, v}$ is laminar. If for some $Y \in \mathcal{L}_{u, \bar{v}}$ the set $\left\{X_{u, v}, Y\right\}$ is not laminar, then the cut $X_{u, v} \cap Y$ may not be an optimal $u-v$ cut because of the definition of $X_{u, v}$. But then $X_{u, v} \cup Y$ is an optimal $u-v$ cut by Claim 12/1. Let

$$
\mathcal{Y}:=\left\{Y: Y \in \mathcal{L}_{u, \bar{v}} \wedge\left\{X_{u, v}, Y\right\} \text { is not laminar }\right\} .
$$

The set $\left\{X_{u, v} \cup Y: Y \in \mathcal{Y}\right\}$ consists of optimal $u-v$ cuts and totally ordered by $\subseteq$. By taking a cofinal sequence of type at most $\omega$ and applying 3 ' we obtain that $X_{0}:=\bigcup \mathcal{Y}$ is an optimal $u-v$ cut. Note that $\left\{X_{0}\right\} \cup\left(\mathcal{L} \backslash \mathcal{L}_{\bar{u}, \bar{v}}\right)$ is laminar. For each $Z \in \mathcal{L}_{\bar{u}, \bar{v}}$ fix some $s_{Z}, t_{Z}$ such that $Z$ is an optimal $s_{Z}-t_{Z}$ cut. We claim that if for such a $Z$ the pair $\left\{X_{0}, Z\right\}$ is not laminar, then $\left\{X_{u, v}, Z\right\}$ is not laminar as well. Indeed, consider just the construction of $X_{0}$ and the fact that if for a cut $Y \in \mathcal{L}_{u, \bar{v}}$ we have $Y \cap Z \neq \varnothing$, then $Z \subseteq Y$ by the laminarity. Let

$$
\mathcal{Z}:=\left\{Z \in \mathcal{L}_{\bar{u}, \bar{v}}:\left\{X_{0}, Z\right\} \text { is not laminar }\right\} .
$$

For $Z \in \mathcal{Z}$ we know that $\left\{X_{u, v}, Z\right\}$ is not laminar. By the definition of $X_{u, v}$ the cut $X_{u, v} \backslash Z$ may not be an optimal $u-v$ cut hence by Claim $12 / 4$ it follows, that $s_{Z} \in X_{u, v}\left(\subseteq X_{0}\right)$. Finally by Claim $12 / 4$ we may take $X^{*}=X \cup \bigcup \mathcal{Z}$ by adding countably many elements of $\mathcal{Z}$ with union $\bigcup \mathcal{Z}$ one by one to $X$ and taking limit.

\section{A counterexample}

In the previous section we obtained (as a special case of Theorem 6) the existence of a laminar system of optimal cuts for countably infinite finitely separable weighted graphs which elements separate any vertex pair optimally. In this section we show by an example that one cannot guarantee the 
existence of a Gomory-Hu tree as well without further assumptions. Let $V=\left\{v_{n}: n \in \mathbb{N}\right\}$ and let $E=\left\{v_{\infty} v_{n}: n \in \mathbb{N}\right\} \cup\left\{v_{n} v_{n+1}: n \in \mathbb{N}\right\}$. Finally $c\left(v_{\infty} v_{n}\right):=1$ for all $n \in \mathbb{N}$ and with the notation $e_{n}:=v_{n} v_{n+1}$

$$
c\left(e_{n}\right):= \begin{cases}2 & \text { if } n=0 \\ c\left(e_{n-1}\right)+n+1 & \text { if } n>0 .\end{cases}
$$

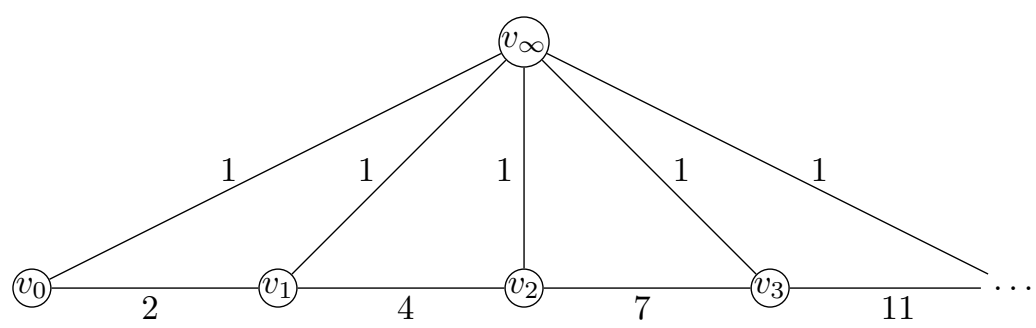

Figure 1: A finitely separable weighted graph without a Gomory-Hu tree

Claim 15. If $n<m$, then $\left\{v_{0}, v_{1}, \ldots, v_{n}\right\}=: V_{n}$ is the only optimal $v_{n}-v_{m}$ cut.

Proof: Pick an optimal $v_{n}-v_{m}$ cut $X$. Since $d_{c}\left(V_{n}\right)<c\left(e_{k}\right)$ whenever $k>n$, a cut $X$ may not separate the end vertices of such an $e_{k}$. Then $v_{\infty} \notin X$ otherwise $d_{c}(X)=\infty$. Thus we have $X \subseteq V_{n}$. Suppose, for seeking a contradiction, that $v_{l} \notin X$ for some $l<n$ and $l$ is the largest such an index. Then

$$
d_{c}(X)-d_{c}\left(V_{n}\right) \geq c\left(e_{l}\right)-l-1>0,
$$

which contradicts to the optimality of $X$.

Claim 16. $(G, c)$ has no Gomory-Hu tree.

Proof: Assume, to the contrary, that $T$ is a Gomory-Hu tree of $(G, c)$. For all $e \in E(T)$ pick the fundamental cut $X_{e}$ that corresponds to $e$ and does not contain $v_{\infty}$. On the one hand, $\mathcal{L}:=$ $\left\{X_{e}\right\}_{e \in E(T)}$ is a laminar system of optimal cuts that contains at least one $\subseteq$-maximal element (if $e$ incident with $v_{\infty}$ in $T$, then $X_{e}$ is a maximal element). On the other hand, $\mathcal{L}=\left\{V_{n}: n \in \mathbb{N}\right\}$ since the optimal cuts are unique up to complementation and the additional condition "does not contain $v_{\infty}$ " makes them unique. This is a contradiction since $\left(V_{n}\right)$ is a strictly increasing sequence.

Remark 17 . One can obtain also a locally finite counterexample by some easy modification of our counterexample above.

\section{Existence of an abstract Gomory-Hu tree}

In this section we prove or main result (which is Theorem 3). It will be convenient to use the following equivalent but formally weaker definition of Gomory-Hu trees.

Claim 18. $T=(V, F)$ is a Gomory-Hu tree with respect to $b$ if for all uv $\in F$ the fundamental cuts corresponding to uv in $T$ separate optimally $u$ and $v$. 
Proof: Let $u \neq v \in V$ be arbitrary and let $v_{1}, v_{2}, \ldots, v_{m}$ be the vertices of the unique $u-v$ path in $T$ numbered in the path order.

Proposition 19. For all pairwise distinct $u, v, w \in V$ we have:

$$
\lambda_{b}(u, w) \geq \min \left\{\lambda_{b}(u, v), \lambda_{b}(v, w)\right\} .
$$

Proof: It follows from the fact that if a cut separates $u$ and $w$, then it separates either $u$ and $v$ or $v$ and $w$ as well.

On one hand by applying the Proposition above repeatedly we obtain

$$
\lambda_{b}(u, v) \geq \min \left\{\lambda_{b}\left(v_{i}, v_{i+1}\right): 1 \leq i<m\right\}=: \lambda_{b}\left(v_{i_{0}}, v_{i_{0}+1}\right) \text { for some } 1 \leq i_{0}<m .
$$

On the other hand, the fundamental cuts corresponding to the edge $v_{i_{0}} v_{i_{0}+1}$ separates $u$ and $v$ and have value $\lambda_{b}\left(v_{i_{0}}, v_{i_{0}+1}\right)$ by assumption. Thus

$$
\lambda_{b}(u, v) \leq \lambda_{b}\left(v_{i_{0}}, v_{i_{0}+1}\right) .
$$

Hence equality holds and the fundamental cuts corresponding to $v_{i_{0}} v_{i_{0}+1} \in F$ are optimal cuts between $u$ and $v$.

A sequence $\left(X_{n}\right)$ of optimal cuts is essential if all of its members separate optimally a vertex pair that the earlier members do not.

Lemma 20. If $\left(X_{n}\right)$ is $a \subseteq$-monotone sequence of optimal cuts and $\lim X_{n}=: X \notin\{\varnothing, V\}$, then $\left(X_{n}\right)$ has no essential subsequence.

Proof: Assume, to the contrary, that $\left(X_{n}\right)$ is a counterexample. By symmetry we may suppose that $\left(X_{n}\right)$ is increasing. By trimming $\left(X_{n}\right)$ we may assume that it is essential witnessed by $s_{n}, t_{n}$ i.e. $X_{n}$ is an optimal $s_{n}-t_{n}$ cut but $X_{m}$ is not whenever $m<n$.

Claim 21. $t_{n} \notin X$ holds for all large enough $n$.

Proof: Suppose that $t_{n} \in X$ for infinitely many $n$. By the monotone-continuity the numerical sequence $\left(b\left(X_{n}\right)\right)$ converges to $b(X)>0$, thus $b\left(X_{n}\right) \geq b(X) / 2>0$ for large enough $n$. On the one hand, $b\left(X \backslash X_{n}\right) \rightarrow 0$ since $\left(X \backslash X_{n}\right) \rightarrow \varnothing$ monotonously. On the other hand, $X \backslash X_{n}$ is a $t_{n}-s_{n}$ cut for infinitely many $n$ because of the indirect assumption and for such an $n$

$$
b\left(X \backslash X_{n}\right) \geq b\left(X_{n}\right) \geq b(X) / 2>0
$$

which is a contradiction.

By trimming $\left(X_{n}\right)$, we may assume that $t_{n} \notin X$ for all $n$. It implies that $b\left(X_{n}\right) \leq b\left(X_{n+1}\right)$ for each $n$ because $X_{n+1}$ is an $s_{n}-t_{n}$ cut and $X_{n}$ is an optimal $s_{n}-t_{n}$ cut. But then $s_{n+1} \notin X_{n}$ for all $n$, otherwise $X_{n}$ would be at least as good $s_{n+1}-t_{t_{n+1}}$ cut as the optimal one but $X_{n+1}$ is the first optimal $s_{n+1}-t_{n+1}$ cut of the sequence by the choice of $\left(X_{n}\right)$.

Claim 22. $X_{n}$ is an optimal $s_{n}-s_{n+1}$ cut for all $n$. 
Proof: By Corollary 13, there is an $Y \in\left\{X_{s_{n}, s_{n+1}}, X_{s_{n+1}, s_{n}}\right\}$ for which $Y \subseteq X_{n+1}$. If $b(Y)<b\left(X_{n}\right)$ would hold, then $Y$ would be either a better $s_{n}-t_{n}$ cut than $X_{n}$ or a better $s_{n+1}-t_{n+1}$ cut than $X_{n+1}$; which both are impossible.

Since $s_{n} \in X$ for al $n$ the Claim above contradicts to Claim 21 with the choices $s_{n}:=s_{n}$ and $t_{n}:=s_{n+1}$.

Take an optimal cut $X$. For $u \neq v \in X$ let $\boldsymbol{u} \prec_{\boldsymbol{X}} \boldsymbol{v}$ if $X_{u, v} \nsubseteq X$.

Claim 23. The relation $\prec_{X}$ is a strict partial ordering on $X$.

Proof: It is irreflexive by definition. For transitivity assume $u \prec_{X} v \prec_{X} w$. If $u=w$, then we have $u \prec_{X} v$ and $v \prec_{X} u$ which contradicts to Corollary 13 . Thus we may assume that $u, v, w$ are pairwise distinct. Suppose, to contrary, that $u \prec_{X} w$ does not hold i.e. $X_{u, w} \subseteq X$. Assume first that $v \in X_{u, w}$. By Corollary 13, either $X_{u, v} \subseteq X_{u, w}$ or $X_{v, u} \subseteq X_{u, w}$. Since $u \prec_{X} v$, necessarily $X_{v, u} \subseteq X_{u, w}$. But then $X_{u, w}$ and $X_{v, u}$ are both $v-w$ cuts and

$$
\lambda_{b}(v, w) \geq \min \left\{\lambda_{b}(v, u), \lambda_{b}(u, w)\right\}=\min \left\{b\left(X_{v, u}\right), b\left(X_{u, w}\right)\right\}
$$

shows that one of them is optimal which contradicts to $v \prec_{X} w$.

Hence $v \notin X_{u, w}$ holds. $X_{u, w}$ is not an optimal $u-v$ cut since $u \prec_{X} v$. Therefore $b\left(X_{u, w}\right)>$ $b\left(X_{v, u}\right)$. (Note that $X_{v, u} \subseteq X$ by $u \prec_{X} v$ and by Corollary 13). Hence $w \notin X_{v, u}$ otherwise $X_{v, u}$ would be a better cut between $w$ and $u$ than the optimal. On the other hand, $X_{v, u}$ is not an optimal $v-w$ cut since $v \prec_{X} w$ hence $X_{w, v} \subseteq X$ and $b\left(X_{w, v}\right)<b\left(X_{v, u}\right)$ hold. Necessarily $u \in X_{w, v}$, otherwise $X_{w, v}$ separates better $w$ and $u$ than $X_{u, w}$, but then $X_{w, v}$ separates better $u$ and $v$ than $X_{v, u}$, which is a contradiction.

Lemma 24. If $X$ is an optimal $s-t$ cut, then $X$ has $a \prec_{X}$-minimal element $s^{\prime}$. For all such an $s^{\prime}$, cut $X$ it is an optimal $s^{\prime}-t$ cut.

Proof: Let $A=\left\{x \in X: \lambda_{b}(x, t)=\lambda_{b}(s, t)\right\}$ and $B:=\left\{y \in X: \lambda_{b}(y, t)<\lambda_{b}(s, t)\right\}$. Then $A \cup B$ is a partition of $X$. Note that $A \neq \varnothing$ since $s \in A$.

Proposition 25. For all $x \in A$ and $y \in B: x \prec_{X} y$ holds.

Proof: If $x \in A$ and $y \in B$, then $\lambda_{b}(x, y)<\lambda_{b}(s, t)\left(=\lambda_{b}(x, t)\right)$, otherwise

$$
\lambda_{b}(y, t) \geq \min \left\{\lambda_{b}(x, y), \lambda_{b}(x, t)\right\}=\lambda_{b}(x, t)=\lambda_{b}(s, t)
$$

contradicts to $y \in B$. Therefore if $X_{x, y} \subseteq X$ would hold, then (since $X_{x, y}$ is a $x$-t cut)

$$
\lambda_{b}(x, t) \leq \lambda_{b}(x, y)<\lambda_{b}(s, t)
$$

which is impossible since $x \in A$.

By Proposition 25, it is enough to find a minimal element for the poset $\left(A, \prec_{X}\right)$. The existence of such an element follows immediately from the following Proposition.

Proposition 26. Set $A$ is finite. 
Proof: Assume, to seeking for contradiction, that $A$ is infinite. Pick a nested sequence $\left(A_{n}\right)$ of nonempty subsets of $A$ with $\bigcap_{n=0}^{\infty} A_{n}=\varnothing$. On the one hand, $b\left(A_{n}\right) \rightarrow 0$ by property 3 . On the other hand, every $A_{n}$ separates an $x \in A$ from $t$ and hence

$$
b\left(A_{n}\right) \geq \lambda_{b}(x, t)=\lambda_{b}(s, t)>0,
$$

which is a contradiction.

For the second part of Lemma 24 let $s^{\prime}$ be a $\prec_{X}$-minimal element of $X$. Then by Proposition $25 s^{\prime} \in A$ thus $\lambda_{b}\left(s^{\prime}, t\right)=\lambda_{b}(s, t)$ by the definition of $A$.

Claim 27. For any $s \in V$ the family $\mathcal{C}_{s}:=\left\{X_{u, s}: u \in V \backslash\{s\}\right\}$ of optimal cuts is laminar.

Proof: Suppose, to the contrary, that $\left\{X_{u, s}, X_{v, s}\right\} \subseteq \mathcal{C}_{s}$ is not laminar. If $u \in X_{v, s}$, then $X_{u, s} \cap X_{v, s}$ is an $u-s$ cut and $X_{u, s} \cup X_{v, s}$ is a $v-s$ cut. By submodularity $X_{u, s} \cap X_{v, s}$ is an optimal $u-s$ cut (and $X_{u, s} \cup X_{v, s}$ is an optimal $v-s$ cut) which contradicts to the definition of $X_{u, s}$. For $v \in X_{u, s}$ the argument is the same. Finally if $u \in X_{u, s} \backslash X_{v, s}$ and $v \in X_{v, s} \backslash X_{u, s}$, then $X_{u, s} \backslash X_{v, s}$ is an $u-s$ cut and $X_{v, s} \backslash X_{u, s}$ is an $v-s$ cut thus by applying Remark 4 follows that they are also optimal, contradicting to the definitions of $X_{v, s}$ and $X_{u, s}$.

Let $\prec_{V}$ be the trivial partial ordering on $V$ (i.e. under which there are no comparable elements).

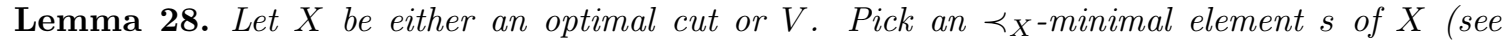
Lemma 24). Then the $\subseteq$-maximal elements of the laminar system $\mathcal{C}_{\boldsymbol{s}, \boldsymbol{X}}:=\left\{X_{u, s}: u \in X \backslash\{s\}\right\}$ forms a partition of $X \backslash\{s\}$.

Proof: By the choice of $s$ we know that $\cup \mathcal{C}_{s, X} \subseteq X \backslash\{s\}$. It is enough to show that for any $u^{*} \in X \backslash\{s\}$ the laminar system $\mathcal{C}_{s, X}$ has a maximal element that contains $u^{*}$. Assume, seeking for contradiction, that it is false and $\left(X_{u_{n}, s}\right)$ is a strictly increasing sequence that shows this. On the one hand, this sequence is essential because $X_{u_{m}, s}$ may not be an optimal $u_{n}-s$ cut for $m<n$ since $X_{u_{n}, s}$ is the $\subseteq$-smallest such a cut. On the other hand, $\lim X_{u_{n}, s} \subseteq V \backslash\{s\}$ which contradicts to Lemma 20.

We build the desired abstract Gomory-Hu tree for $b$ by using Lemma 28 repeatedly. Pick an arbitrary $r \in V$ for root. It makes possible to define a unique fundamental cut for each edge $e$ of the tree, namely the vertex set of the component after deletion of $e$ that does not contain $r$. Let $\left\{X_{i}\right\}_{i \in I_{0}}$ consists of the maximal elements of the laminar system $\mathcal{C}_{r}$. Let $x_{i}$ be a $\prec_{X_{i}}$-minimal element of $X_{i}$ and draw the tree-edges $r x_{i}$ for $i \in I_{0}$. Note that Lemma 24 ensures that the fundamental cut corresponds to $r x_{i}$ will separate optimally $r$ and $x_{i}$ assuming that $X_{i}$ will be the vertex set of the subtree rooted at $x_{i}$. Take now for each $i \in I_{0}$ the $\subseteq$-maximal elements $\left\{X_{i, j}\right\}_{j \in I_{1}}$ of $\mathcal{C}_{x_{i}, X_{i}}$ and choose a $\prec_{X_{i, j}}$-minimal element $x_{i, j}$ of $X_{i, j}$. Draw the tree-edges $x_{i} x_{i, j}$ for all $i \in I_{0}$ and $j \in I_{1}$. By continuing recursively the process we claim that every $v \in V$ has to appear in the tree. Indeed, if some $v$ does not, then we would obtain a nested essential sequence of optimal cuts such that its limit contains $v$ which contradicts to Lemma 20.

\section{References}

[1] Dunwoody, M. Structure trees and networks. arXiv preprint arXiv:1311.3929 (2013). 
[2] Evangelidou, A., And Papasoglu, P. A cactus theorem for end cuts. International Journal of Algebra and Computation 24, 01 (2014), 95-112.

[3] Gomory, R. E., And Hu, T. C. Multi-terminal network flows. Journal of the Society for Industrial and Applied Mathematics 9, 4 (1961), 551-570. 